\title{
The XN-30 hematology analyzer for rapid sensitive detection of malaria: a diagnostic accuracy study
}

Annelies Post ${ }^{1 * \dagger} \mathbb{B}$, Berenger Kaboré ${ }^{1,2+} \mathbb{D}$, Isaie J. Reuling ${ }^{3+} \mathbb{B}$, Joel Bognini ${ }^{2}$, Wouter van der Heijden ${ }^{1}$, Salou Diallo², Palpouguini Lompo ${ }^{2}$, Basile Kam², Natacha Herssens ${ }^{4}$, Kjerstin Lanke $^{3}$, Teun Bousema ${ }^{3}$, Robert W. Sauerwein ${ }^{3}$ (D) Halidou Tinto ${ }^{2,5,6}$ (D) Jan Jacobs ${ }^{4,7}$, Quirijn de Mast ${ }^{1}$ (D) and Andre J. van der Ven ${ }^{1 *}$ (D)

\begin{abstract}
Background: Accurate and timely diagnosis of malaria is essential for disease management and surveillance. Thin and thick blood smear microscopy and malaria rapid diagnostic tests (RDTs) are standard malaria diagnostics, but both methods have limitations. The novel automated hematology analyzer $\mathrm{XN}$-30 provides standard complete blood counts (CBC) as well as quantification of malaria parasitemia at the price of a CBC. This study assessed the accuracy of XN-30 for malaria detection in a controlled human malaria infection (CHMI) study and a phase 3 diagnostic accuracy study in Burkina Faso.
\end{abstract}

Methods: Sixteen healthy, malaria-naive CHMI participants were challenged with five Plasmodium falciparum-infected mosquitoes. Blood was sampled daily for XN-30, blood smear microscopy, and malaria qPCR. The accuracy study included patients aged $>3$ months presenting with acute febrile illness. XN-30, microscopy, and rapid diagnostic tests (HRP-2/pLDH) were performed on site; qPCR was done in retrospect. The malaria reference standard was microscopy, and results were corrected for sub-microscopic cases.

Results: All CHMI participants became parasitemic by qPCR and XN-30 with a strong correlation for parasite density $\left(R^{2}=0.91 ; p<.0001\right)$. The XN-30 accurately monitored treatment and allowed detection of recrudescence. Out of 908 patients in the accuracy study, 241 had microscopic malaria (density 24-491,802 parasites/ $\mu \mathrm{L}$ ). The sensitivity and specificity of XN-30 compared to microscopy were $98.7 \%$ and $99.4 \%$ (PPV $=98.7 \%, \mathrm{NPV}=99.4 \%$ ). Results were corrected for qPCR-confirmed sub-microscopic cases. Three microscopy-confirmed cases were not detected by XN-30. However, XN-30 detected 19/134 (14.2\%) qPCR-confirmed cases missed by microscopy. Among qPCR-confirmed cases, XN-30 had a higher sensitivity (70.9\% versus 66.4\%; $p=.0009)$ and similar specificity (99.6\% versus $100 \% ; p=.5)$ as microscopy. The accuracy of XN-30 for microscopic malaria was equal to or higher than HRP-2 and pLDH RDTs, respectively.

Conclusions: The XN-30 is a novel, automated hematology analyzer that combines standard hemocytometry with rapid, objective, and robust malaria detection and quantification, ensuring prompt treatment of malaria and malaria anemia and follow-up of treatment response.

Trial registration: Both trials were registered on clinicaltrials.gov with respective identifiers NCT02836002 (CHMI trial) and NCT02669823 (diagnostic accuracy study).

Keywords: Malaria, Diagnosis, Sensitivity, Specificity, Burkina Faso

\footnotetext{
* Correspondence: annelies.post@gmail.com;

andre.vanderven@radboudumc.nl

${ }^{\dagger}$ Annelies Post, Berenger Kaboré and Isaie J. Reuling contributed equally to

this work.

${ }^{1}$ Nijmegen Institute of International Health, Radboud University Medical

Centre, Nijmegen, the Netherlands

Full list of author information is available at the end of the article
}

(c) The Author(s). 2019 Open Access This article is distributed under the terms of the Creative Commons Attribution 4.0 International License (http://creativecommons.org/licenses/by/4.0/), which permits unrestricted use, distribution, and reproduction in any medium, provided you give appropriate credit to the original author(s) and the source, provide a link to the Creative Commons license, and indicate if changes were made. The Creative Commons Public Domain Dedication waiver (http://creativecommons.org/publicdomain/zero/1.0/) applies to the data made available in this article, unless otherwise stated. 


\section{Background}

Malaria remains a major cause of morbidity and mortality around the world. Sub-Saharan Africa is most affected with Plasmodium falciparum accounting for 99\% of estimated cases [1]. Timely and accurate diagnosis of malaria is essential for disease management and control [2]. Thin and thick blood smear microscopy (further referred to as microscopy) and malaria rapid diagnostic tests (RDTs) are standard malaria diagnostics in endemic areas. RDTs have significantly improved the use of diagnostics for malaria diagnosis, accounting for $74 \%$ of diagnostic tests performed among suspected cases in 2015 [3]. Both techniques provide challenges in clinical practice: RDTs are antigen-directed and relative to their design, can neither quantify malaria parasitemia, nor allow monitoring of treatment response, prerequisites to manage severe malaria. Additionally, RDTs cannot distinguish current from recently treated infections [4]. Consequently, false-positive results may be misinterpreted as treatment failure [5]. WHO therefore reserves a role for microscopy, which is however labor-intensive and its quality is heavily observer-dependent. Molecular tests are complex to perform, require highly trained personnel, and are relatively expensive, limiting its general use.

The XN-30 (Sysmex, Kobe, Japan) is a novel automated hematology analyzer and malaria diagnostic that directly detects and quantifies Plasmodium parasites (falciparum and non-falciparum) in blood using violet laser technology [6-8]. Our study aimed to evaluate the accuracy of the XN-30 for the detection of Plasmodium falciparum malaria. First, we assessed its performance in healthy participants in whom low-density parasitemia was induced in a controlled human malaria infection (CHMI) study. To establish the performance under field conditions, we then performed a phase 3 diagnostic accuracy study among febrile patients in Burkina Faso.

\section{Methods}

\section{Controlled human malaria infection (CHMI)}

The ability of the XN-30 to detect low-density sexual and asexual parasitemia and monitoring of treatment was studied in a CHMI trial (ClinicalTrials.gov, NCT02836002). Details and results of the primary objectives of this study have recently been published [9]. Ethylenediaminetetraacetic acid (EDTA)-anticoagulated venous blood was collected twice daily for $\mathrm{XN}-30$, and results were compared with qPCR for asexual- and qRT-PCR for sexual P. falciparum parasites. Both techniques are described in detail elsewhere [9].

\section{Diagnostic accuracy study}

This prospective, double-blinded, phase 3 diagnostic accuracy study (ClinicalTrials.gov, NCT02669823) was performed at the Clinical Research Unit of Nanoro (CRUN) [10]. The primary objectives were (i) to assess the diagnostic sensitivity and specificity of the XN-30 to detect malaria parasitemia in children and adults with an acute febrile illness against thick blood smear microscopy or qPCR in case of a negative thick smear or incongruent results of $\mathrm{XN}-30$ and microscopy and (ii) to assess the diagnostic accuracy of the Infection Manager System (IMS) for the detection of bacterial bloodstream infection in a malaria-endemic area. The secondary objective was to compare the accuracy of the XN-30 analyzer to diagnose malaria compared to malaria RDT. The results of the IMS are reported elsewhere to increase legibility.

\section{Procedures}

Nanoro is a rural area of Burkina Faso which is hyperendemic for Plasmodium falciparum, though Plasmodium ovale and Plasmodium malariae are sporadically found [11]. Participants were enrolled between March 2016 and June 2017 at the "Centre Medicale avec Antenne Chirurgicale" (CMA) of Nanoro, to which CRUN is affiliated. Consecutive patients of 3 months and older suspected of acute febrile illness were screened for eligibility. Patients were eligible if they had a measured temperature of $\geq 38.0^{\circ}$ $\mathrm{C}$ or $\leq 35.5^{\circ} \mathrm{C}$, or a reported history of fever up to $48 \mathrm{~h}$ prior to presentation, or suspicion of severe infection with signs of severe clinical illness. Patients with fever lasting more than 7 days were excluded. Upon inclusion, 2-5 mL EDTA anti-coagulated blood was obtained and analyzed within $1 \mathrm{~h}$ after sampling. Laboratory analyses were performed and interpreted by experienced laboratory technicians who were blinded to clinical data. Patients were followed daily during hospitalization, and follow-up samples were taken if clinically indicated. Another follow-up sample was taken at approximately 2 weeks after inclusion.

Data was collected on case report forms (CRFs) and entered into a secure database (RedCap, Vanderbilt University, Nashville, USA) after conformity check by a medical doctor. Entered data were checked against the CRFs by a data manager. Approximately $10 \%$ of participant study files were checked by an independent monitor. Results from qPCR were entered into an Excel (Microsoft, Washington, USA) database and merged with the principal database. The researchers were blinded to the $\mathrm{XN}-30$ until the clinical database was locked. Interpretation of the index test was done by blinded researchers after inclusion was completed. Laboratory procedures were standardized; quality controls were performed according to good clinical and laboratory practices (GCLP) guidelines. 


\section{Index test: $\mathrm{XN}-30$}

The $\mathrm{XN}-30$ is an automated hematology analyzer (Sysmex Corporation, Kobe, Japan) which can be used for malaria detection as described in detail elsewhere [6]. The analyzer aspirates and dilutes blood samples in a diluent solution (CELLPACK DCL). Subsequently, the nucleic acids are stained with a staining solution (Fluorocell $\mathrm{M}$ ) along with a lysis solution (LysercellM). Infected red blood cells (iRBC) and white blood cells (WBC) are detected by a violet semiconductor $405 \mathrm{~nm}$ laser beam. Parasitemia percentage is calculated by the ratio of infectedand uninfected red blood cells. Output data separately reports a complete blood count $(\mathrm{CBC})$, the presence of gametocytes and parasitemia-both as a percentage of infected red blood cells (MI-RBC\%) and absolute parasite density (MI-RBC\#) expressed as parasites $/ \mu \mathrm{L}$. The data is automatically determined by analyzing scatter grams plotted three dimensionally with forward-side scattered light, side scattered light, and side fluorescent light. iRBC are visible as a separate $\mathrm{RBC}$ population on the scattergram (Fig. 1). In case of abnormal scatter gram distribution, the malaria result is reported as "inconclusive."
The analyzer aspirates approximately $60 \mu \mathrm{L}$ whole blood per analysis; results are available within $1 \mathrm{~min}$. $\mathrm{XN}-30$ will be made for global distribution, and the price of a measurement is expected to be comparable to regular hemocytometry [6]). No formal quantification limit was applied for $\mathrm{XN}-30$ for the CHMI study. In the diagnostic accuracy study, XN-30 values of $\geq 20 \mathrm{MI}-\mathrm{RBC} / \mu \mathrm{L}$ with at least $5 \%$ ring stages were considered positive. Validated hemocytometry data were used for clinical care, all experimental data were kept blinded.

\section{Reference tests: malaria diagnostics}

Both RDT and microscopy were performed using EDTA-anticoagulated blood. Thick and thin blood films were examined for the presence of Plasmodium parasites according to WHO procedures [12]. Results were expressed as asexual parasites per microliter using the patients' WBC count. The Plasmodium species and presence of gametocytes were also recorded. Slides were viewed by two independent microscopists. Presence of one or more Plasmodium parasites was considered positive. In case of discrepancies, the result of a third microscopist was decisive.
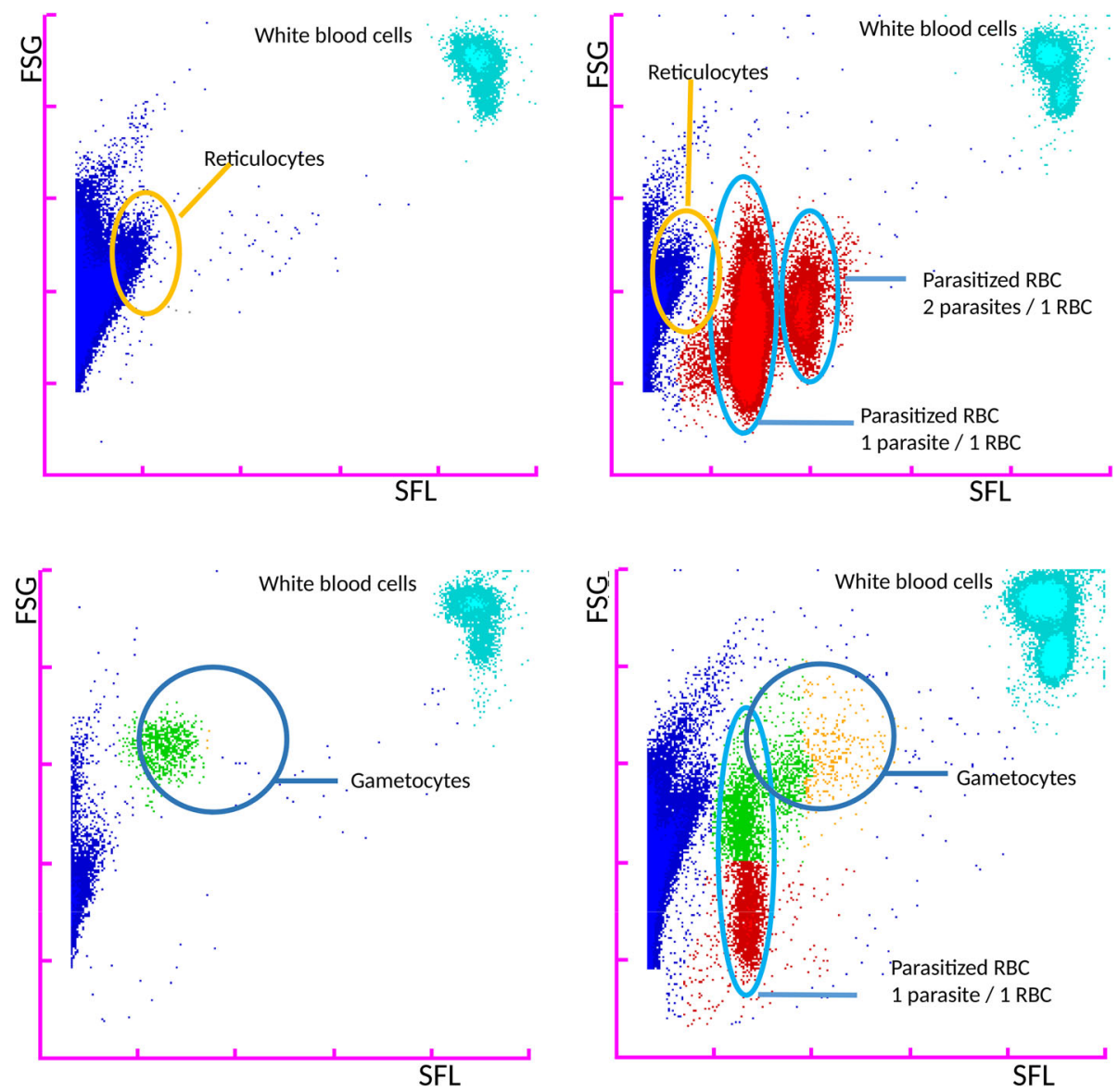

Fig. 1 Forward sideward scatter of patient with and without malaria and gametocytes as recorded by the XN-30 

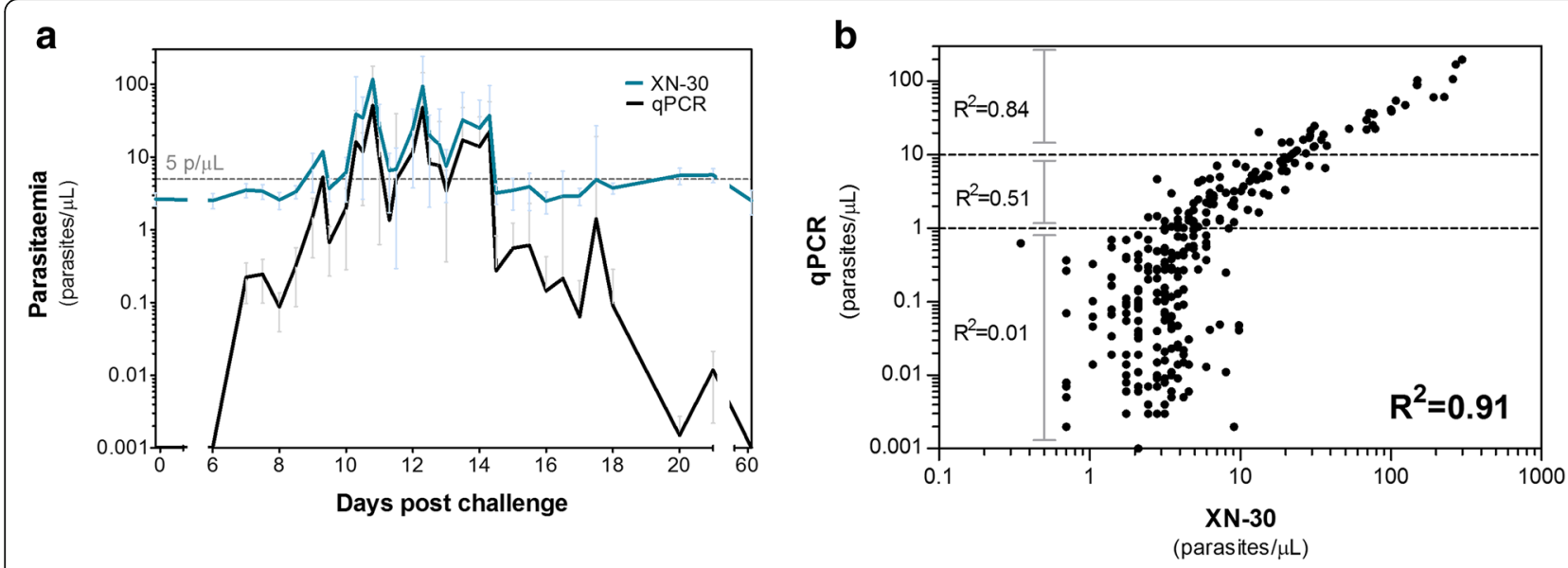

Fig. 2 Diagnostic performance of $\mathrm{XN}-30$ in $\mathrm{CHMl}$ model expressed as (a) line graph and (b) scatterplot

SD Bioline Malaria Ag-P.f (HRP2/pLDH) (Standard Diagnostics, Inc., Gyeonggi-do, Republic of Korea) was performed according to the manufacturer's instructions. Five microliters of venous blood was inoculated on the RDT cassette. After 5 and $15 \mathrm{~min}$, the test line reactivity of the Plasmodium falciparum histidine-rich protein-2 (HRP-2) and pan-Plasmodium species parasite-lactate dehydrogenase (pLDH) were scored, whereby any visible test line was considered positive. Results for pLDH and HRP-2 were recorded separately. Quantitative PCR (qPCR) targeting the multicopy $18 \mathrm{~S}$ rRNA gene was performed in retrospect at the Department of Microbiology of Radboud University Medical Centre, the Netherlands, using $200 \mu \mathrm{L}$ blood per patient using previously
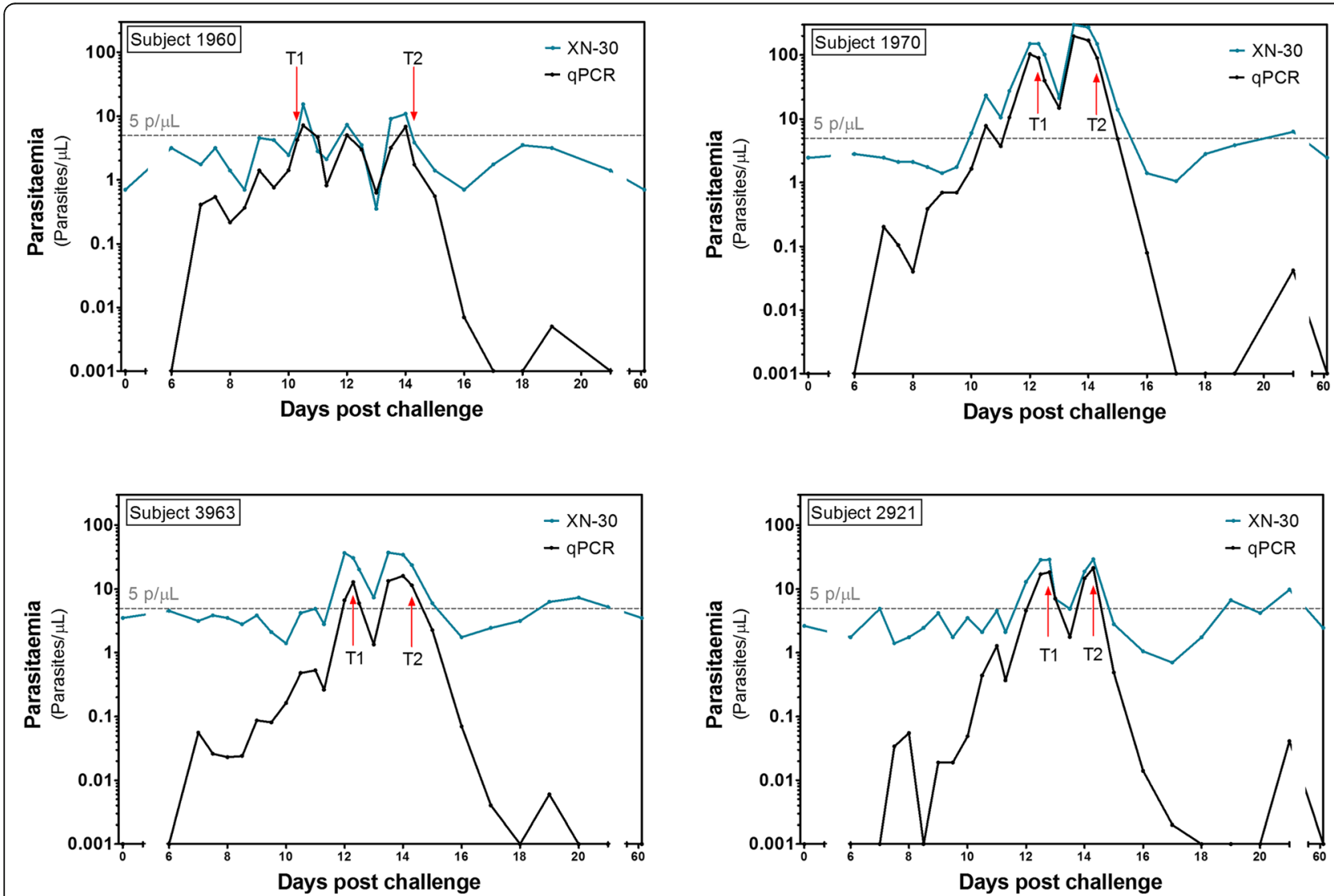

Fig. 3 Parasite density curves of participants with recrudescent infections. The parasite density treatment threshold of $5 \mathrm{p} / \mathrm{uL}$ is indicated with a grey line 
described methods [13]. The lower level of detection of qPCR was 0.05 parasites per microliter $(\mathrm{p} / \mu \mathrm{L})$.

\section{Case definitions, data management, and statistical analysis}

Malaria parasitemia was defined as the presence of one or more parasites in the asexual stage in malaria microscopy or a malaria qPCR result over $0.05 \mathrm{p} / \mu \mathrm{L}$ in the diagnostic accuracy study. In case only gametocytes were observed, microscopy was considered negative. Severe malaria was defined according to WHO criteria 2014 [14].

A statistical plan was made before data inspection. Analyses were done using Stata 14 (Stata Corp, College Station, TX, USA). Patients with missing data on either reference test, index test, or qPCR were excluded from the analysis. In the case of inconclusive index test results due to an abnormal scattergram distribution, the result was reported as "inconclusive." Differences in proportions, medians, or means were compared using respectively the chi-square test, two-tailed Fisher's exact test, or Mann-Whitney $U$ test in case of not normally distributed data. Sensitivity, specificity, positive predictive value (PPV), and negative predictive value (NPV) were assessed using the STATA diagt-package. A receiver operation characteristics (ROC) curve analysis was done to assess the area under the curve (AUC). Linear regression analyses were done to assess the correlation in parasite densities between qPCR, malaria thick smear, and $\mathrm{XN}-30$. Comparative analyses of sensitivity and specificity were done using a McNemar test and reported as test ratio with significance level. A significance level of $5 \%$ was used for all analyses.

\section{Results}

\section{Controlled human malaria infection}

We assessed the diagnostic accuracy of $\mathrm{XN}-30$ in 16 healthy participants exposed to low-density parasitemia during a CHMI (Additional file 3: Figure S1). All participants developed parasitemia between day 6 and 12

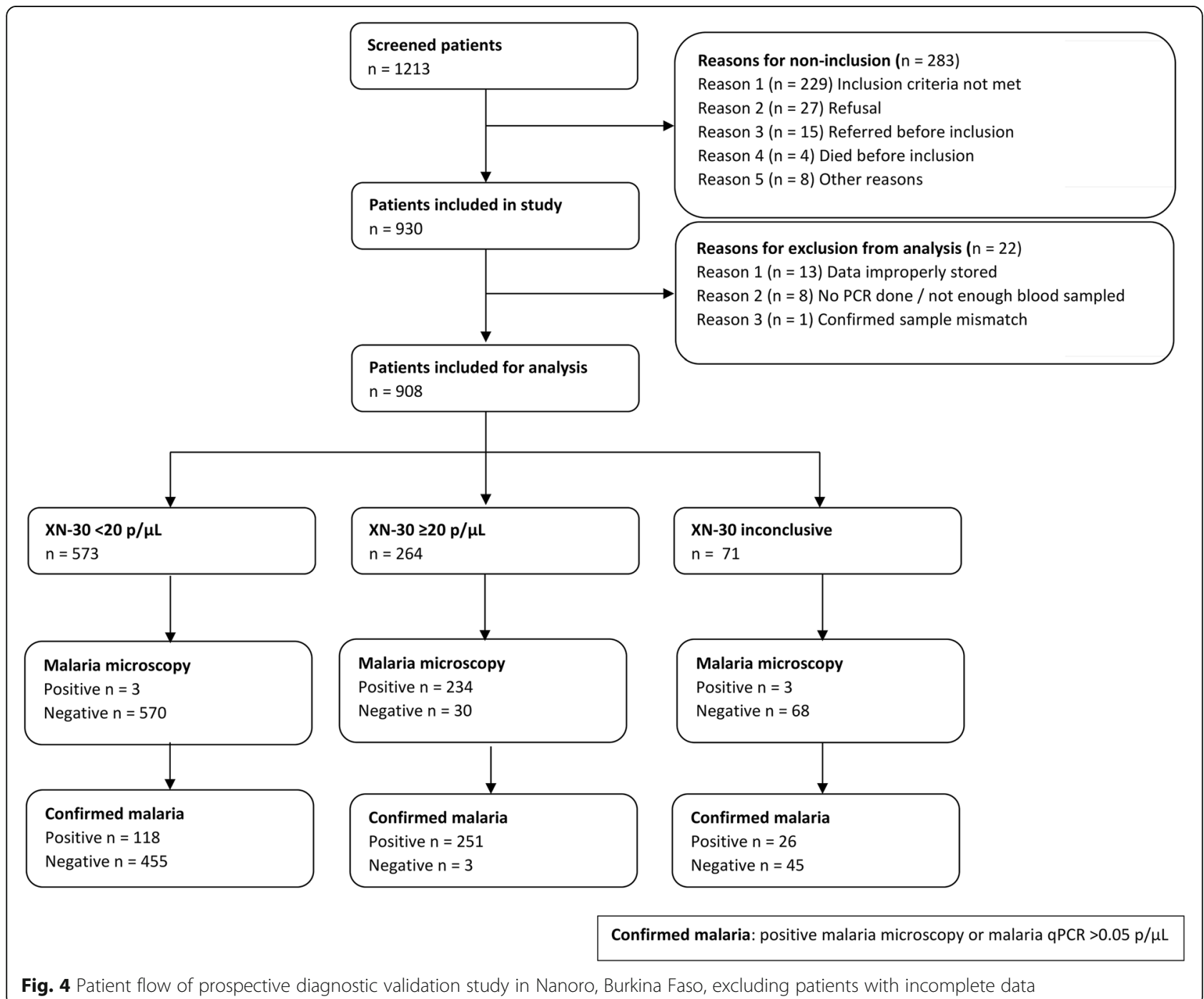


post-infection, as determined by qPCR. The geometric mean peak parasitemia density was $21 \mathrm{p} / \mu \mathrm{L}$. Figure $2 \mathrm{a}$ shows parasitemia dynamics over time as quantified by the XN-30 analyzer and by qPCR. The quantitative data shows a strong correlation between the $\mathrm{XN}-30$ and $\mathrm{qPCR}\left(R^{2}=0.91 ; p<.0001\right)$ (Fig. $\left.2 \mathrm{~b}\right)$. The XN-30 was able to monitor treatment and detect recrudescent infections (Fig. 3). The positive cutoff of the $\mathrm{XN}-30$ was $8.1 \mathrm{p} / \mu \mathrm{L}$ as defined by the mean of all negative qPCR samples +3 SD. Gametocyte densities in CHMI were too low to be detected by microscopy or $\mathrm{XN}-30$ (< 1.3 gametocytes/ $\mu \mathrm{L})$. In vitro experiments were performed to estimate the limit of detection of mature gametocytes and test the stability of the samples when measured by XN-30 (Additional file 4: Figure S2 and Additional file 6: Figure S4). Sample quantification remained accurate up to $6 \mathrm{~h}$ after sampling, at room temperature (Additional file 5: Figure S3).

\section{Diagnostic accuracy study}

We assessed the diagnostic accuracy under field conditions in a malaria hyperendemic region in Burkina Faso. A total of 1213 eligible patients presented at CMA Nanoro, of whom 930 were included (Fig. 4). Fourteen patients were excluded from analysis due to inadequate sample storage or evident sample mismatch. For eight patients, the PCR data was not available due to a shortage of sampled blood. Additionally, in 71 patients (7.8\%), the XN-30 scattergram was abnormally distributed and therefore reported as "inconclusive." There were significantly more patients aged $0-2$ and patients with malnutrition among this group. Malaria was significantly less prevalent (Additional file 1: Table S1).

Baseline characteristics for all included patients are presented by age group in Table 1 . The percentage of patients who were pre-treated with antimalarials prior to admission ranged between $25.8 \%(84 / 335)$ in adults and $46.1 \%(83 / 180)$ in children $2-5$ years old. Severe malaria

Table 1 Baseline characteristics $(n=908)$

\begin{tabular}{|c|c|c|c|c|}
\hline & \multicolumn{3}{|l|}{ Children } & \multirow{3}{*}{$\begin{array}{l}\text { Adults } \\
>15 \text { years } \\
n=335\end{array}$} \\
\hline & $0-2$ years & $2-5$ years & $5-15$ years & \\
\hline & $n=266$ & $n=180$ & $n=127$ & \\
\hline & Median (IQR) & Median (IQR) & Median (IQR) & Median (IQR) \\
\hline Age distribution (months, years) & $12(8-17)$ months & 36 (28-46) months & $8(6-10)$ years & $41(29-59)$ years \\
\hline Male: female ratio & 1.5 & 1.5 & 1.3 & 1.2 \\
\hline Fever (days) & $3(2-3)$ & $3(2-3)$ & $3(2-4)$ & $3(2-4)$ \\
\hline Temperature $\left({ }^{\circ} \mathrm{C}\right)$ & $38.5(38.0-39.4)$ & $38.3(37.9-39.4)$ & $38.2(37.7-39.0)$ & $38.3(38.0-39.0)$ \\
\hline Systolic BP (mm/Hg) & $98(89-106)$ & $98(90-108)$ & $102(95-112)$ & $108(96-124)$ \\
\hline Diastolic BP (mm/Hg) & $61(53-65)$ & $62(56-69)$ & $63(59-75)$ & $66(60-76)$ \\
\hline MAP $(\mathrm{mm} / \mathrm{Hg})$ & $73(66-79)$ & $75(67-81)$ & $80(72-87)$ & $83(72-92)$ \\
\hline Pulse (min) & $125(112-133)$ & $123(108-135)$ & $116(100-125)$ & $104(96-119)$ \\
\hline Respiratory rate (min) & $35(32-42)$ & $32(30-38)$ & $29(26-30)$ & $27(26-28)$ \\
\hline Hemoglobin (g/dL) & $7.8(5.6-10.0)$ & $8.6(6.5-10.7)$ & $9.4(7.8-11.6)$ & $10.3(8.4-12.8)$ \\
\hline WBC $\left(\right.$ cells $\left.\times 10^{3} / \mu \mathrm{L}\right)$ & $13.1(10.0-19.4)$ & $11.2(7.9-16.5)$. & $10.6(7.4-16.6)$ & $7.9(5.5-12.8)$ \\
\hline \multirow[t]{2}{*}{ Platelets (cells $\times 10^{3} / \mu \mathrm{L}$ ) } & $277(124-452)$ & $232(126-358)$ & $271(181-372)$ & $237(156-337)$ \\
\hline & $n(\%)$ & $n(\%)$ & $n(\%)$ & $n(\%)$ \\
\hline Antimalarials taken in past 2 weeks & $93(34.2)$ & $83(46.1)$ & $54(42.1)$ & $86(25.8)$ \\
\hline HIV (confirmed before inclusion) & $1(0.4)$ & $2(1.1)$ & $3(2.3)$ & $11(3.3)$ \\
\hline Malnourished ${ }^{1}$ & $112(43.4)$ & $70(41.2)$ & $47(41.6)$ & $84(28.6)$ \\
\hline Severe anaemia ${ }^{2}$ & $87(32.7)$ & $50(27.8)$ & $33(25.8)$ & $72(27.7)$ \\
\hline Severe malaria & $56(21.0)$ & $47(26.1)$ & $12(9.3)$ & $5(1.5)$ \\
\hline Severe anemia & $28(50.0)$ & $15(31.9)$ & $8(66.7)$ & $4(80.0)$ \\
\hline Cerebral malaria & $8(14.3)$ & $10(21.3)$ & $3(25.0)$ & 0 \\
\hline Respiratory distress & $2(3.6)$ & $4(8.5)$ & 0 & 0 \\
\hline Parasitemia > $150.000 \mathrm{p} / \mu \mathrm{L}$ & $18(32.1)$ & $18(38.3)$ & $1(8.3)$ & $1(20.0)$ \\
\hline
\end{tabular}

$B P$ blood pressure, MAP mean arterial pressure, WBC white blood cells

${ }^{1}$ Based on Z-scores for children $<5$ and BMI for older patients, data available for respectively $258,170,113$, and 294 patients per age group

${ }^{2}$ Based on WHO criteria for severe anemia 
was detected in 120 (50.0\%) patients with microscopically confirmed malaria, of whom 55 (45.6\%) fulfilled the criteria of severe anemia. These high numbers are in line with previously reported results [11] and are likely caused by the function of CMA as a district referral hospital for complicated disease. In total, 411 follow-up samples were taken: 59 during hospitalization and 352 at 2-week follow-up.

The results of malaria diagnostics are presented in Table 2. A total of 240 patients (26.4\%) had positive microscopy with parasite densities ranging from 24 to $500,000 \mathrm{p} / \mu \mathrm{L}$ (median 15,614; IQR 745-76,901); 374 patients $(41.2 \%)$ had a positive qPCR. Sub-microscopic parasitemia (positive qPCR with negative microscopy) was detected in 134 patients $(14.8 \%)$ with densities ranging from 0.05 to $102.8 \mathrm{p} / \mu \mathrm{L}$ (median 0.68 ; IQR 0.13 3.1). Six microscopy-positive cases were not detected by qPCR. RDTs were positive in 396 patients; in more than half of the cases, both pLDH and HRP-2 were positive. XN-30 recorded 255 cases of malaria with a median parasite density of $12,390 \mathrm{p} / \mu \mathrm{L}$ (IQR 650 $88,656)$.

Tables 3 and 4 present the diagnostic sensitivity, specificity, PPV, and NPV for XN-30 against microscopy and qPCR. Figure 5 shows the corresponding ROC curves at the cutoff value of $20 \mathrm{p} / \mu \mathrm{L}$. The sensitivity and specificity of XN-30 against microscopy were $98.7 \%$ and $96.5 \%$, respectively. In 21 cases, the $\mathrm{XN}-30$ indicated parasitemia whereas microscopy was deemed negative; 19/21 cases were later confirmed as sub-microscopic parasitemia by qPCR. Three samples were false negatives: two cases had low parasite densities $(<20 \mathrm{p} / \mu \mathrm{L}$ in $\mathrm{qPCR})$, the third had a high parasite density and was suspected to be a sample mix-up based on discrepancies in CBC between the $\mathrm{XN}-30$ and a second hematology analyzer used for clinical care. After correction for sub-microscopic malaria, the sensitivity and specificity of the XN-30 increased to 98.8\% and $99.7 \%$ respectively with a PPV of $99.2 \%$ and an NPV of 99.5\% (AUC 0.99).

Table 4 presents the diagnostic accuracy for $\mathrm{XN}-30$ and microscopy compared to qPCR. The XN-30 had a significantly higher sensitivity than microscopy to detect qPCR-confirmed parasitemia (70.9\% versus 66.4\%, $p=.0009)$ whereas specificity did not differ between both tests $(99.6 \%$ versus $100 \%, p=.5$ ). The cutoff value of best fit to $\mathrm{qPCR}$ in the current dataset was a parasite density of $26 \mathrm{p} / \mu \mathrm{L}$, which gave similar results to the proposed cutoff value (sensitivity 69.5\%, specificity 98.8\%) (Additional file 2: Table S2, Additional file 6: Figure S4).

A secondary objective was to compare the diagnostic performance of $\mathrm{XN}-30$ to diagnose malaria parasitemia against two RDTs. Table 5 shows the diagnostic accuracy of both used RDTs and XN-30 compared to uncorrected microscopy (upper part) and qPCR (lower part). Considering all qPCR-confirmed cases, the sensitivity of XN-30 was significantly higher than $\mathrm{pLDH}(70.9 \%$ versus $60.2 \%$, $p<.0001$ ), while specificity was equal (both $99.6 \%, p=$ 1.0). Conversely, $\mathrm{XN}-30$ had higher specificity $(99.6 \%$ versus $87.7 \%, p<.0001$ ) than HRP-2, while the sensitivity of HRP-2 was higher than that of $\mathrm{XN}-30$ (86.3\% versus $70.9 \%, p<.001)$. The difference in sensitivity between HRP-2 and XN-30 was mostly caused by sub-microscopic parasitemia cases (median parasite density $0.45 \mathrm{p} / \mu \mathrm{L}$; IQR $0.13-1.8)$, most of whom (64.0\%) had been pre-treated with antimalarials. This suggests that at least part of the cases detected by HRP-2, but missed by XN-30, may have

Table 2 Cases of malaria detected per type of diagnostic, for all included patients $(n=916)$

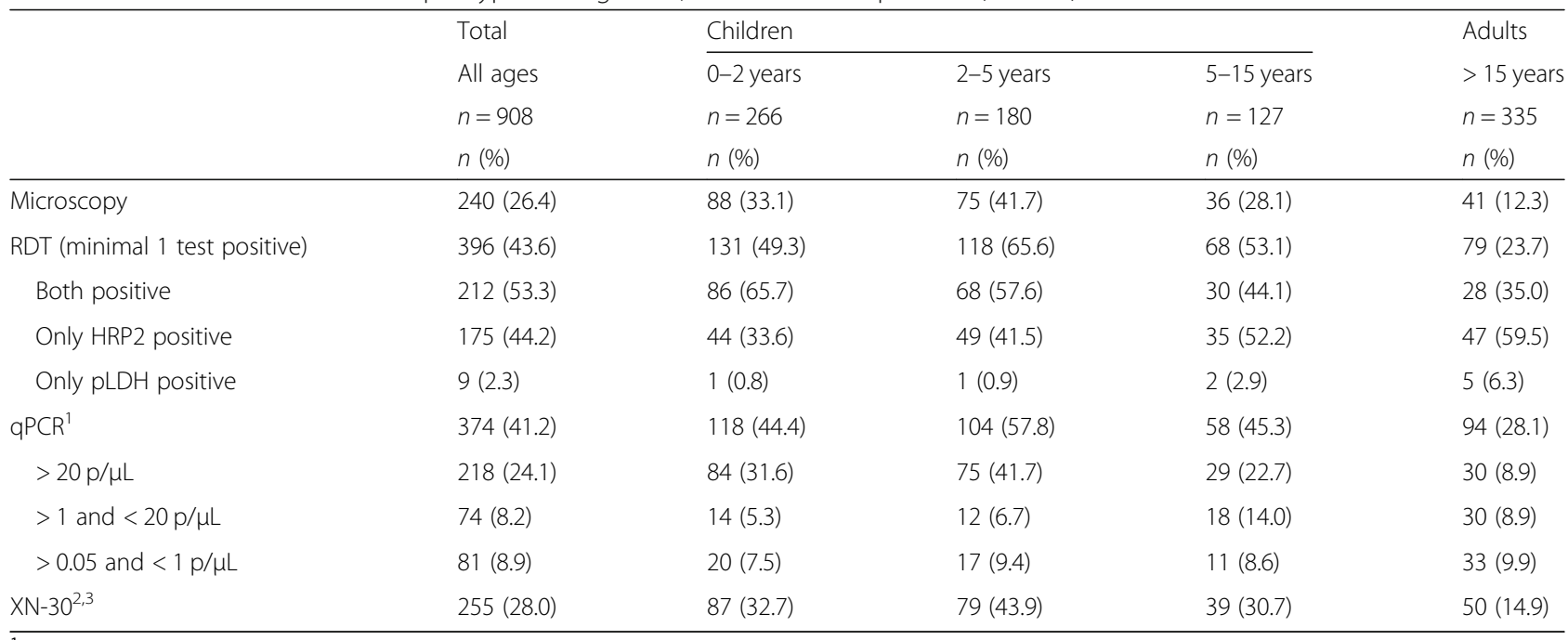

${ }^{1}$ Excluding six false negative PCRs, which were most likely false negative due to sample mix-up

${ }^{2}$ Excluding 71 patients with an abnormal scattergram, three of whom had positive malaria microscopy

${ }^{3}$ There were three false negative cases detected by microscopy but not detected by $\mathrm{XN}-30$ 
Table 3 Diagnostic accuracy of XN-30 compared to malaria microscopy. Data with and without correction for qPCR confirmed submicroscopic parasitemia cases

\begin{tabular}{|c|c|c|c|c|}
\hline & \multicolumn{2}{|c|}{$\mathrm{XN}-30$, uncorrected $(n=837)$} & \multicolumn{2}{|c|}{$\mathrm{XN}-30$, corrected ${ }^{*}(n=837)$} \\
\hline & Positive & Negative & Positive & Negative \\
\hline Positive microscopy & 234 & 3 & 253 & 3 \\
\hline Negative microscopy & 21 & 579 & 2 & 579 \\
\hline Sensitivity & $98.7 \%$ & & $98.8 \%$ & \\
\hline Specificity & $96.5 \%$ & & $99.7 \%$ & \\
\hline PPV & $91.8 \%$ & & $99.2 \%$ & \\
\hline NPV & $99.5 \%$ & & $99.5 \%$ & \\
\hline ROC & 0.98 & & 0.99 & \\
\hline
\end{tabular}

*In case of incongruent results between malaria microscopy and $\mathrm{XN}-30$, the microscopy data was adjusted to the PCR result

been based on remaining presence of antigens after a recent malaria infection. Among microscopically confirmed cases, $\mathrm{XN}-30$ had an equal or higher sensitivity and higher specificity than HRP-2 and pLDH.

We compared parasite densities as recorded by XN-30 with densities quantified by microscopy and qPCR. Figure 6 shows the corresponding correlations (microscopy $r=0.82, p<.0001 ;$ qPCR $r=0.79, p<.0001$ ). Follow-up samples were available in 125 patients $(51.9 \%)$ with $\mathrm{XN}$-30-confirmed malaria. The median parasite density dropped from 19,138 p/ $\mu \mathrm{L}$ (IQR 1594-98,165) at inclusion to below the limit of positivity $(20 \mathrm{p} / \mu \mathrm{L})$ at follow-up, confirming that $\mathrm{XN}-30$ can be used for treatment follow-up in a clinical setting. A total of 15 cases of non-falciparum malaria parasitemia were identified by microscopy, all of which were picked up by $\mathrm{XN}-30$, though they were not differentiated from $P$. falciparum.

\section{Discussion}

This is the first prospective clinical study to present the diagnostic performance of XN-30. The sensitivity of $\mathrm{XN}-30$ to detect Plasmodium falciparum parasitemia was superior to that of microscopy in qPCR-confirmed cases, whereas the specificity was comparable. $\mathrm{XN}-30$ had an equal or higher sensitivity than HRP-2 and pLDH respectively, and a higher specificity than both for microscopy-confirmed malaria. Our results are in line with results from a recently published retrospective study on the performance of the XN-30 from South Africa [15]. The accuracy of malaria microscopy (LOD 24 $\mathrm{p} / \mu \mathrm{L}$ ) in the current study is far above the accuracy of microscopy under field conditions, which is estimated at $50-100 \mathrm{p} / \mu \mathrm{L}$ [16]. The reported accuracy of $\mathrm{XN}-30$ might therefore be an under-estimation compared to most clinical settings. Commonly used RDTs have a detection limit around $100-200 \mathrm{p} / \mu \mathrm{L}[17,18]$, except when the HRP-2 deletion of P. falciparum, as found in Central Africa, is present that prevents the detection of this strain [19].

In case of signs of severe malaria, follow-up of admitted malaria patients, or RDT-confirmed malaria cases with persistent fever despite anti-malarial treatment, the WHO recommends the use of malaria microscopy [20]. Our data suggest that XN-30 could be used as an alternative microscopy in laboratories where hematology analyzers are already used. XN-30 shares many of the advantages of microscopy, including relatively low costs, quantification of parasite

Table 4 Prevalence of disease on qPCR, N (\%, 95\% Cl) *McNemar test to compare sensitivity and specificity present. All ratios presented as $\mathrm{XN}-30$ relative to microscopy

\begin{tabular}{|c|c|c|c|c|c|c|}
\hline & \multicolumn{6}{|c|}{$\mathrm{XN}-30$ and microscopy versus qPCR results $(n=837)$} \\
\hline & \multicolumn{2}{|l|}{$\mathrm{XN}-30$} & \multicolumn{2}{|c|}{ Microscopy } & \multirow[t]{2}{*}{ Test ratio $(95 \% \mathrm{Cl})$} & \multirow[t]{2}{*}{$p$ value } \\
\hline & Positive & Negative & Positive & Negative & & \\
\hline $\mathrm{qPCR}>0.05 \mathrm{p} / \mu \mathrm{L}$ & 253 & 104 & 237 & 120 & & \\
\hline $\mathrm{qPCR}<0.05 \mathrm{p} / \mu \mathrm{L}$ & 2 & 478 & 0 & 480 & & \\
\hline Sensitivity & $70.9 \%$ & & $66.4 \%$ & & $0.94(0.90-0.97)$ & .0009 \\
\hline Specificity & $99.6 \%$ & & $100.0 \%$ & & 0 & .5 \\
\hline PPV & $98.9 \%$ & & $100.0 \%$ & & & \\
\hline NPV & $83.4 \%$ & & $80.0 \%$ & & & \\
\hline ROC & 0.86 & & 0.83 & & & \\
\hline
\end{tabular}

$\overline{q P C R}$ qualitative polymerase chain reaction, $P P V$ positive predictive value, $N P V$ negative predictive value 

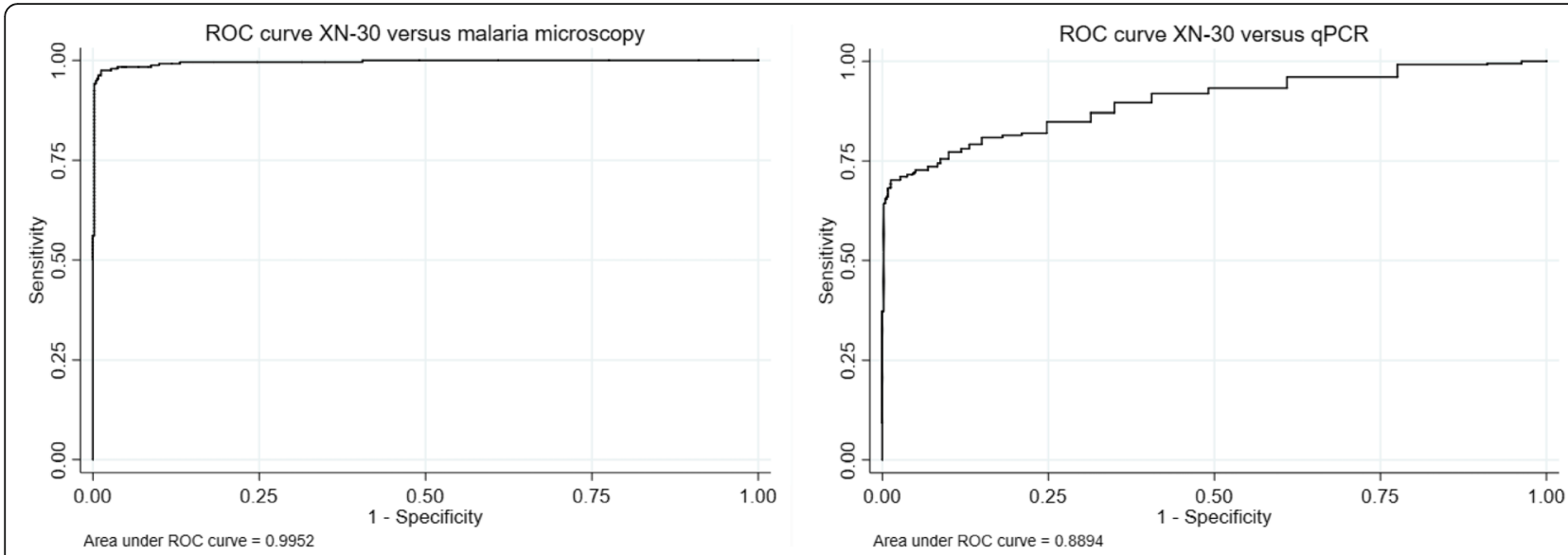

Fig. 5 ROC curves $X N-30$ versus malaria microscopy and $q P C R$

density, and monitoring of treatment response. However, in contrast to microscopy, $\mathrm{XN}-30$ can be used by minimally trained staff, without sample preparation, has no observer-dependent viability, and is available around-the-clock, whereas trained malaria microscopists are often limitedly available. Another important additional advantage of the $\mathrm{XN}-30$ is that it simultaneously provides a $\mathrm{CBC}$ with each analysis and therefore synchronizes malaria diagnose and management. Severe malaria anemia is a major cause of death, and a delay of blood transfusion is associated with poor outcome [21].

Automated hematology analyzers to diagnose malaria have been studied in the last two decades, applying different techniques such as abnormal depolarization in the granularity of phagocytes, abnormalities in the size of blood cells, or blood cell differentiation patterns [22-27]. However, these methods often carry limitations such as low- and variable sensitivities and specificities due to technical limitations and confounding

Table 5 Prevalence of disease on microscopy (upper part of the table) and qPCR (lower part of the table), N (\%, 95\% Cl) * McNemar to compare sensitivity and specificity present ratio of proportions. All ratios presented as XN-30 relative to HRP-2 and pLDH respectively

\begin{tabular}{|c|c|c|c|c|c|c|c|c|c|c|}
\hline \multicolumn{11}{|c|}{ XN-30 and RDT (HRP-2 and pLDH) versus corrected microscopy results ( $n=837)$} \\
\hline & \multicolumn{2}{|l|}{$\mathrm{XN}-30$} & \multicolumn{2}{|l|}{ HRP-2 } & \multirow{2}{*}{$\begin{array}{l}\text { Test ratio } \\
95 \% \mathrm{Cl}\end{array}$} & \multirow[t]{2}{*}{$p$ value } & \multicolumn{2}{|l|}{$\mathrm{pLDH}$} & \multirow{2}{*}{$\begin{array}{l}\text { Test ratio } \\
95 \% \mathrm{Cl}\end{array}$} & \multirow[t]{2}{*}{$p$ value } \\
\hline & Pos & Neg & Pos & Neg & & & Pos & Neg & & \\
\hline Microscopy positive & 234 & 3 & 231 & 6 & & & 208 & 29 & & \\
\hline Microscopy negative & 21 & 579 & 136 & 464 & & & 9 & 591 & & \\
\hline Sensitivity & $98.7 \%$ & & $97.5 \%$ & & $0.99(0.97-1.01)$ & $p=.45$ & $87.8 \%$ & & $0.89(0.85-0.93)$ & $p<.0001$ \\
\hline Specificity & $96.5 \%$ & & $77.3 \%$ & & $6.47(4.31-9.73)$ & $p<.0001$ & $98.5 \%$ & & $0.43(0.23-0.81)$ & $p=.012$ \\
\hline PPV & $91.8 \%$ & & $62.9 \%$ & & & & $95.9 \%$ & & & \\
\hline NPV & $99.5 \%$ & & $98.7 \%$ & & & & $95.3 \%$ & & & \\
\hline \multicolumn{11}{|c|}{ XN-30 and RDT (HRP-2 and pLDH) versus qPCR results $(n=837)$} \\
\hline & $\mathrm{XN}-30$ & & HRP-2 & & Test ratio & $p$ value & $\mathrm{pLDH}$ & & Test ratio & $p$ value \\
\hline & Pos & Neg & Pos & Neg & $95 \% \mathrm{Cl}$ & & Pos & Neg & $95 \% \mathrm{Cl}$ & \\
\hline $\mathrm{qPCR}>0.05 \mathrm{p} / \mu \mathrm{L}$ & 253 & 104 & 308 & 49 & & & 215 & 142 & & \\
\hline $\mathrm{qPCR}<0.05 \mathrm{p} / \mathrm{\mu L}$ & 2 & 478 & 59 & 421 & & & 2 & 478 & & \\
\hline Sensitivity & $70.9 \%$ & & $86.3 \%$ & & $1.22(1.15-1.29)$ & $<0.0001$ & $60.2 \%$ & & $0.85(0.80-0.89)$ & $<.0001$ \\
\hline Specificity & $99.6 \%$ & & $87.7 \%$ & & $0.04(0.01-0.14)$ & $<0.0001$ & $99.6 \%$ & & $1.0(0.07-13.8)$ & 1.0 \\
\hline PPV & $99.2 \%$ & & $83.9 \%$ & & & & $99.1 \%$ & & & \\
\hline NPV & $82.1 \%$ & & $89.6 \%$ & & & & $77.1 \%$ & & & \\
\hline
\end{tabular}

Pos positive, neg negative, $H R P 2$ histidine-rich protein $2, p L D H$ pan-Plasmodium lactate dehydrogenase, $q P C R$ qualitative polymerase chain reaction, $P P V$ positive predictive value, NPV negative predictive value 


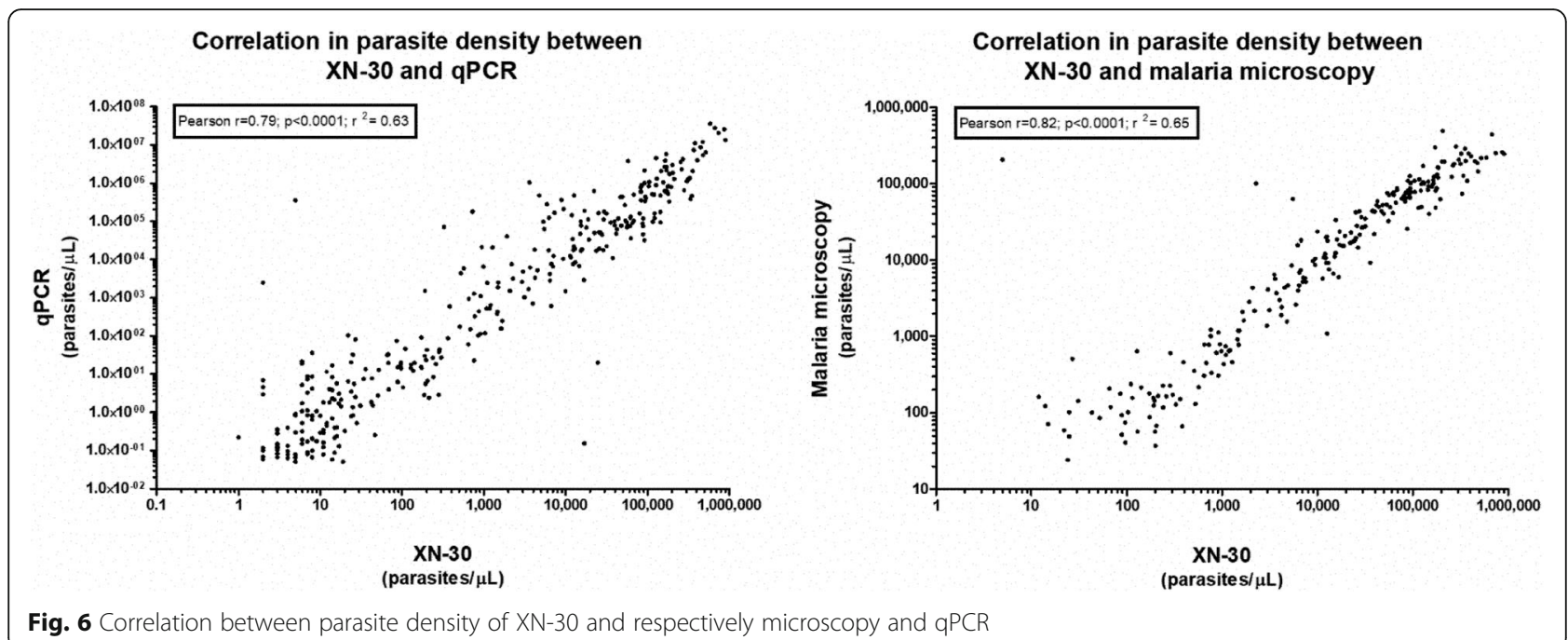

host- and pathology-specific differences [27, 28]. Direct staining of malaria nucleic acid in parasites and subsequent analysis by a flow cytometry-like technique may prevent drawbacks from indirect tests [29-31]. Several studies have demonstrated that iRBC can be detected through pigmented DNA or RNA strands [32-34]. The XN-30 combines both techniques in a fully automated manner. Since the analyzer can be connected to WiFi, data could be instantaneously linked to any organization involved in epidemiological research and disease control.

The limit of detection as observed in the CHMI study was lower than that of the diagnostic accuracy study (8.1 versus $20 \mathrm{p} / \mu \mathrm{L}$ ). We have no obvious explanation for this difference; previous studies demonstrate that malaria negative samples with low hemoglobin, high reticulocyte count, thrombocytopenia or hemoglobinopathies had no significant influence on the reliability of the malaria result [8]. Our data as presented in additional Table 1 suggests that this may occur more frequently in very young children and patients with malnutrition, but further research is needed to analyze the origin of this difference.

\section{Limitations}

In the present study, $\mathrm{XN}-30$ had an inconclusive result due to an abnormal scatter gram distribution in 71 cases (7.8\%). This implies that when used in clinical settings, approximately one in 12 patients would need an additional malaria diagnostic when the $\mathrm{XN}-30$ is inconclusive. Second, the $\mathrm{XN}-30$ currently does not reliably distinguish Plasmodium species prevalent in Burkina Faso. In addition, this study was performed in a malaria hyperendemic setting; therefore, the population-level effect of the sensitivity and specificity could vary by different settings e.g. low-endemicity countries.

\section{Conclusion}

In conclusion, the $\mathrm{XN}-30$ holds promise as a rapid and sensitive test for malaria detection and subsequent treatment monitoring. Since XN-30 provides a CBC with each analysis, it provides critical information for malaria management at a price comparable to $\mathrm{CBC}$. Its higher sensitivity compared to microscopy in low-density parasitemia also makes it a useful tool for mass screening in control programs.

\section{Additional files}

Additional file 1: Table S1. Characteristics of patients with inconclusive XN-30 result. (DOCX $18 \mathrm{~kb}$ )

Additional file 2: Table S2. ROC analysis of cutoff value of best fit, XN30 compared to qPCR. (DOCX $20 \mathrm{~kb}$ )

Additional file 3: Figure S1. CHMl study participant inclusion flow. (TIF $73 \mathrm{~kb}$ )

Additional file 4: Figure S2. Correlation of the XN-30 with microscopy and $\mathrm{gPCR}$, in cultured parasites. (TIF $660 \mathrm{~kb}$ )

Additional file 5: Figure S3. Stability data of cultured ring-stage samples. (TIF 181 kb)

Additional file 6: Figure S4. ROC curve at cutoff value of best fit, $\mathrm{XN}$-30 compared to qPCR. (TIF $1006 \mathrm{~kb}$ )

\section{Abbreviations}

AUC: Area under the curve; CBC: Complete blood count; CHMI: Controlled human malaria infection; CMA: Centre Medicale avec Antenne Chirurgicale (District Hospital of Nanoro); CRF: Case report form; CRUN: Clinical Research Unit of Nanoro; DNA: Deoxyribonucleic acid;

EDTA: Ethylenediaminetetraacetic acid; GCLP: Good clinical and laboratory practices; HRP2: Histidine-rich protein-2; IMS: Infection Manager System; iRBC: Infected red blood cells; LOD: Limit of detection; MI-RBC(\#): Outcome measure of XN-30: absolute parasite density expressed as parasites $/ \mu \mathrm{L} ; \mathrm{Ml}-$ $\mathrm{RBC}(\%)$ : Outcome measure of XN-30: parasites as a percentage of infected red blood cells; N: Absolute number; NPV: Negative predictive value; $\mathrm{p} /$ $\mu \mathrm{L}$ : Parasites/microliter; pLDH: pan-Plasmodium species parasite-lactate dehydrogenase; PPV: Positive predictive value; qPCR: Quantitative polymerase chain reaction; RBC: Red blood cells; RDT: Rapid diagnostic test; RNA: Ribonucleic acid; ROC: Receiver operation characteristics curve; WBC: White blood cells; WHO: World Health Organization 


\section{Acknowledgements}

The authors would like to thank the nurses from CMA, the laboratory technicians from CRUN, the team of data managers from CRUN, and the study nurses from CRUN-Clement Zongo, Bakombania Abassiri, Catherine Nikiema, Esther Kapioko, and Celine Nare for their dedication to the study. We furthermore thank all study participants for their participation.

\section{Funding}

This work was supported by PATH MVI (CHMI study) and Sysmex Europe $\mathrm{GmbH}$. Sysmex Europe $\mathrm{GmbH}$ provided the analyzer and reagents for the CHMl study and provided full funding for the diagnostic accuracy study. The funding sources were involved in the study design, but not in data collection, analysis and interpretation, or the preparation of the report.

\section{Availability of data and materials}

The datasets used and/or analyzed during the current study are available from the corresponding author on reasonable request.

\section{Authors' contributions}

IjR, WvdH, KL, TB, and RWS were responsible for study design and execution of the CHMI study. AP, BKé, PL, HT, JJ, QdM, and AvV were involved in the study design of the diagnostic accuracy study. BKé, JB, SD, PL, and BKm were responsible for study management in Burkina Faso. NH, AP, and JJ were involved in quality control. Analyses and writing were done by AP, BKé, ljR, QdM, and AvV. All authors read and approved the report before submission.

\section{Ethics approval and consent to participate}

The studies were performed in accordance with the declaration of Helsinki and GCLP guidelines. The CHMI study was approved by the central committee for research involving human subjects of the Netherlands (NL56659.091.16), and the Western Institutional Review Board (WIRB) in the USA. The diagnostic study was approved by the national ethical committee of Burkina Faso (ref 2015-01006), the internal review board of IRSS (ref A03-2016/CEIRES), the ethical committee of the university hospital of Antwerp (ref 15/47/492), and the institutional review board of the Institute of Tropical Medicine Antwerp (ref 1029/15).

\section{Consent for publication}

Not applicable

\section{Competing interests}

AvV and QdM have a non-restricted research grant from SYSMEX, which funded the current study. None of the other authors had any competing interest.

\section{Publisher's Note}

Springer Nature remains neutral with regard to jurisdictional claims in published maps and institutional affiliations.

\section{Author details \\ 'Nijmegen Institute of International Health, Radboud University Medical Centre, Nijmegen, the Netherlands. ${ }^{2}$ RSSS/Clinical Research Unit of Nanoro (CRUN), Nanoro, Burkina Faso. ${ }^{3}$ Department of Medical Microbiology, Radboud University Medical Centre, Nijmegen, the Netherlands. ${ }^{4}$ Department of Clinical Sciences, Institute of Tropical Medicine (ITM), Antwerp, Belgium. ${ }^{5}$ Centre Muraz, Bobo-Dioulasso, Burkina Faso. ${ }^{6}$ Institut Supérieur des Sciences de la Santé, Université Nazi Boni de Bobo-Dioulasso, Bobo-Dioulasso, Burkina Faso. ${ }^{7}$ Department of Microbiology and Immunology, University of Leuven (KU Leuven), Leuven, Belgium.}

Received: 19 January 2019 Accepted: 29 April 2019

Published online: 31 May 2019

\section{References}

1. World Health Organisation: World Malaria Report 2017. 2017.

2. World Health Organisation: A framework for malaria elimination. 2017.

3. World Health Organisation. World Malaria Report 2016. Geneva: World Health Organization; 2016.

4. labal J, Siddique A, Jameel M, Hira PR. Persistent histidine-rich protein 2, parasite lactate dehydrogenase, and panmalarial antigen reactivity after clearance of Plasmodium falciparum monoinfection. J Clin Microbiol. 2004; 42(9):4237-41.
5. McMorrow ML, Aidoo M, Kachur SP. Malaria rapid diagnostic tests in elimination settings--can they find the last parasite? Clin Microbiol Infect. 2011;17(11):1624-31.

6. Tougan T, Suzuki Y, Itagaki S, Izuka M, Toya Y, Uchihashi K, Horii T. An automated haematology analyzer $\mathrm{XN}$-30 distinguishes developmental stages of falciparum malaria parasite cultured in vitro. Malar J. 2018;17(1):59

7. Tougan T, Suzuki Y, Izuka M, Aono K, Okazaki T, Toya Y, Uchihashi K, Horii T. Application of the automated haematology analyzer $\mathrm{XN}-30$ in an experimental rodent model of malaria. Malar J. 2018;17(1):165

8. Pillay $\mathrm{E}$, Litshie $\mathrm{M}$, Coetzer TL. A new era in malaria diagnosis and surveillance using an automated analyzer. Blood. 2017;130(Suppl 1):2097.

9. Reuling IJ, van de Schans LA, Coffeng LE, Lanke K, Meerstein-Kessel L, Graumans W, van Gemert GJ, Teelen K, Siebelink-Stoter R, van de VegteBolmer $\mathrm{M}$, et al. A randomized feasibility trial comparing four antimalarial drug regimens to induce Plasmodium falciparum gametocytemia in the controlled human malaria infection model. eLife. 2018;7. https://doi.org/10. 7554/eLife.31549.

10. Derra K, Rouamba E, Kazienga A, Ouedraogo S, Tahita MC, Sorgho H, Valea I, Tinto H. Profile: Nanoro health and demographic surveillance system. Int J Epidemiol. 2012;41(5):1293-301.

11. Maltha J, Guiraud I, Kaboré B, Lompo P, Ley B, Bottieau E, Van Geet C, Tinto $\mathrm{H}$, Jacobs J. Frequency of severe malaria and invasive bacterial infections among children admitted to a rural hospital in Burkina Faso. PLoS One. 2014;9(2):e89103.

12. World Health Organisation: Basic Malaria Microscopy - Learner's guide, second edition. ISBN 9789241547826 (Part 1). 2010; Available from: https://apps.who.int/iris/bitstream/handle/10665/44208/9789241547826_ eng.pdf;jsessionid=6B258FB78B6EB249A26262660F6150D3? sequence $=1$.

13. Hermsen CC, Telgt DS, Linders EH, van de Locht LA, Eling WM, Mensink EJ, Sauerwein RW. Detection of Plasmodium falciparum malaria parasites in vivo by real-time quantitative PCR. Mol Biochem Parasitol. 2001;118(2): 247-51.

14. World Health Organisation. Severe Malaria. Tropical Med Int Health. 2014;19: 7-131.

15. Pillay E, Khodaiji S, Bezuidenhout BC, Litshie M, Coetzer TL. Evaluation of automated malaria diagnosis using the Sysmex XN-30 analyser in a clinical setting. Malar J. 2019;18(1):15.

16. Ochola LB, Vounatsou $\mathrm{P}$, Smith $\mathrm{T}$, Mabaso MLH, Newton C. The reliability of diagnostic techniques in the diagnosis and management of malaria in the absence of a gold standard. Lancet Infect Dis. 2006;6(9):582-8.

17. Maltha J, Guiraud I, Lompo P, Kabore B, Gillet P, Van Geet C, Tinto H, Jacobs J. Accuracy of PfHRP2 versus Pf-pLDH antigen detection by malaria rapid diagnostic tests in hospitalized children in a seasonal hyperendemic malaria transmission area in Burkina Faso. Malar J. 2014;13:20.

18. Jimenez A, Rees-Channer RR, Perera R, Gamboa D, Chiodini PL, Gonzalez IJ, Mayor A, Ding XC. Analytical sensitivity of current best-in-class malaria rapid diagnostic tests. Malar J. 2017;16(1):128.

19. Cunningham J: Update on Plasmodium falciparum HRP 2/3 gene deletions. Global Malaria Programme. 2018. Available from: https://www.who.int/ malaria/mpac/mpac-mar2017-hrp2-3-deletions-session7-presentation.pdf.

20. World Health Organisation: Universal access to malaria diagnostic testing An operational manual. ISBN 978924150209 2; 2013. Available from: https://apps.who.int/iris/bitstream/handle/10665/44657/9789241502092 eng.pdf?sequence $=1$.

21. Thomas J, Ayieko P, Ogero M, Gachau S, Makone B, Nyachiro W, Mbevi G, Chepkirui M, Malla L, Oliwa J, et al. Blood transfusion delay and outcome in county hospitals in Kenya. Am J Trop Med Hyg. 2017;96(2):511-7.

22. Yoo JH, Song J, Lee KA, Sun YK, Kim YA, Park TS, Choi JR. Automated detection of malaria-associated pseudoeosinophilia and abnormal WBC scattergram by the Sysmex XE-2100 hematology analyzer: a clinical study with 1,801 patients and real-time quantitative PCR analysis in vivax malariaendemic area. Ame J Trop Med Hyg. 2010;82(3):412-4.

23. Wernli M, Tichelli A, von Planta M, Gratwohl A, Speck B. Flow cytometric monitoring of parasitaemia during treatment of severe malaria by exchange transfusion. Eur J Haematol. 1991;46(2):121-3.

24. van Vianen $\mathrm{PH}$, van Engen $\mathrm{A}$, Thaithong $\mathrm{S}$, van der Keur M, Tanke HJ, van der Kaay HJ, Mons B, Janse CJ. Flow cytometric screening of blood samples for malaria parasites. Cytometry. 1993;14(3):276-80.

25. Sharma P, Bhargava M, Sukhachev D, Datta S, Wattal C. LH750 hematology analyzers to identify malaria and dengue and distinguish them from other febrile illnesses. Int J Lab Hematol. 2014;36(1):45-55. 
26. Huh HJ, Oh GY, Huh JW, Chae SL. Malaria detection with the Sysmex XE2100 hematology analyzer using pseudoeosinophilia and abnormal WBC scattergram. Ann Hematol. 2008;87(9):755.

27. Dumas C, Bienvenu AL, Girard S, Picot S, Debize G, Durand B. Automated Plasmodium detection by the Sysmex XN hematology analyzer. J Clin Pathol. 2018;71(7):594-9.

28. Campuzano-Zuluaga G, Hänscheid T, Grobusch MP. Automated haematology analysis to diagnose malaria. Malar J. 2010;9:346.

29. Bianco AE, Battye FL, Brown GV. Plasmodium falciparum: rapid quantification of parasitemia in fixed malaria cultures by flow cytometry. Exp Parasitol. 1986;62(2):275-82.

30. Howard RJ, Battye FL, Mitchell GF. Plasmodium-infected blood cells analyzed and sorted by flow fluorimetry with the deoxyribonucleic acid binding dye 33258 Hoechst. J Histochem Cytochem. 1979;27(4):803-13.

31. Grimberg BT. Methodology and application of flow cytometry for investigation of human malaria parasites. J Immunol Methods. 2011;367:12):1-16.

32. Hare JD. Two-color flow-cytometric analysis of the growth cycle of Plasmodium falciparum in vitro: identification of cell cycle compartments. J Histochem Cytochem. 1986;34(12):1651-8.

33. Mendelow BV, Lyons C, Nhlangothi P, Tana M, Munster M, Wypkema E, Liebowitz L, Marshall L, Scott S, Coetzer TL. Automated malaria detection by depolarization of laser light. Br J Haematol. 1999;104(3):499-503.

34. Janse CJ van Vianen PH, Tanke HJ, Mons B, Ponnudurai T, Overdulve JP. Plasmodium species: flow cytometry and microfluorometry assessments of DNA content and synthesis. Exp Parasitol. 1987;64(1):88-94.

Ready to submit your research? Choose BMC and benefit from:

- fast, convenient online submission

- thorough peer review by experienced researchers in your field

- rapid publication on acceptance

- support for research data, including large and complex data types

- gold Open Access which fosters wider collaboration and increased citations

- maximum visibility for your research: over $100 \mathrm{M}$ website views per year

At BMC, research is always in progress.

Learn more biomedcentral.com/submissions 